\title{
Online Multi-Layers Social Distance Detection in Iraqi Schools
}

\author{
Abdulhakeem Q. Albayati \\ Computer Enhineering Department \\ University of Technology,Iraq
}

Farah F. Alkhalid

Control and Systems Engineering Department

University of Technology,Iraq

\section{Rafah K. Hussain}

Electromechanical Engineering Department

University of Technology,Iraq

Article History: Received: 10 November 2020; Revised 12 January 2021 Accepted: 27 January 2021; Published online: 5 April 2021

\begin{abstract}
In the circumstance of the COVID-19 pandemic, Prevention is better than cure, especially if the cure is not available, the first motto that all health organizations recommend is keep distances between people to prevent epidemic spread. In this paper, an online multi layers social distance detection system is proposed, the main idea is to detect distance among pupils and classify the distance to accept or not, this system treats stream video of fixed camera which monitor the whole school yard where the pupils are available, this proposed system used multi layers, the first is to make person detection using Yolo-4 approach including CNN model, and surround it by rectangle, the second is to specify the center of detected person, finally, calculate the relative distance to decide if it is accepted or not, this system works online and give high accuracy.
\end{abstract}

Keywords: COVID19, Deep Learning, YOLO, CNN

\section{Introduction}

COVID-19 belongs to the family of coronavirus caused diseases, initially reported at Wuhan, China, during late December 2020. On eleventh of March, it spread over 114 countries with 118,000 positive cases and 4000 deaths, WHO declared this a pandemic [1], assist police by minimizing their physical surveillance work in containment zones and public areas, at the same time, in the middle of 2020, many countries allowed people to do their daily activity by keeping care conditions, the first care condition is keep social distance among people to avoid spreading virus.

There are many previous valuable researches, studies, projects and tutorials focused on monitoring, tracking, object detection, in this section, some articles are interested with COVID-19 social distance will be highlighted depending on different approaches.

Authors in [2] studied the relation among social distancing measures and the spread of the COVID-19 epidemic. Authors studies synthetic location specific contact forms to simulate the ongoing path of the outbreak using susceptible-exposed-infected-removed (SEIR) models 
The authors in [3] showed how can social distance among people reduce the mean daily growth rate of COVID-19 in United States (US), supposed a cumulative epidemic size of 4,125 reported cases raised to 26,281 reported cases with no social distancing versus 24,625 reported cases with social distancing within few days.

Shashi Yadav [4] presented a utilizing MobileNet V2 architecture to avoid the spread of the COVID19 virus using camera feeds with raspberry pi4 in real-time.

Singh Punn et al. [5] suggested a real-time using deep learning for monitoring social-distancing utilizing different object detection approaches Faster RCNN, SSD, and YOLO v3. Also, Ramadass and et. al. [6] checked the social distance among people using drone depending on YOLO3. In general YOLO3 or any other version is used for high speed and real-time recognition for many different objects [7] as will be shown later in details.

Rinkal and Ninad [8] studied if people are keeping social distancing by their self-model named SocialdistancingNet-19, and named the detected persons as saved or not,

In general, object detection is the most popular approach that be interested when thinking of image processing, however, YOLOs in different versions are the most familiar.

\section{Social Distance}

Also called physical distancing, the main idea is if people are not close together, they cannot spread germs, viruses and infection. By referring to the official website of the World Health Organization, the social distancing allowed to limit the spread of COVID-19 infection is one meter as minimum [1] as shown in figure 1 . Therefore, the most popular recommendations for all people to keep safety distance among them to prevent spread of disease.

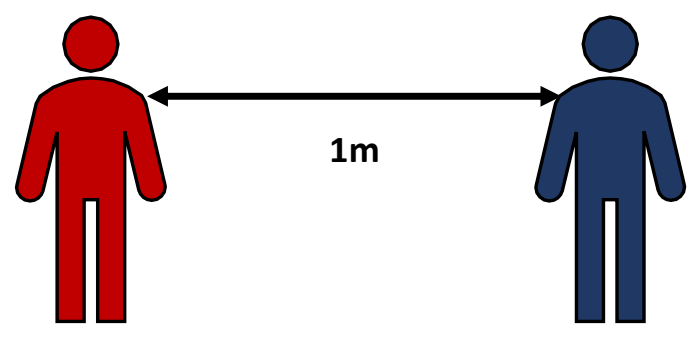

Figure 1. Social distance

\section{Problem Statement}

The pupils are ready to join the new study year, but the fair of spreading COVID-19 is still established, which spreads mainly among people who are in close contact, however, the whole world recommend with keep distances, in this research anew suggestion for surveillance system based on deep learning and object detection in order to alarm monitor if there are contacts less than the social distance.

\section{Proposed Solution}

AS denoted in block diagram in figure 2, the system is started within video stream camera which is fixed in main area to capture many frames. The system will process the captured frame and make sure if there are persons inside, then calculate the distance between each adjacent person and decide if it is accepted or not, then make green rectangle around the two adjacent persons to classify distance is accepted, and red one if not.

The steps in details are listed below:

- Detects people in video streams.

- Detects center.

- Detects full-fledged social distancing.

- Classify the distance:

- Red for not allowed distance.

- Green for allowed distance. 


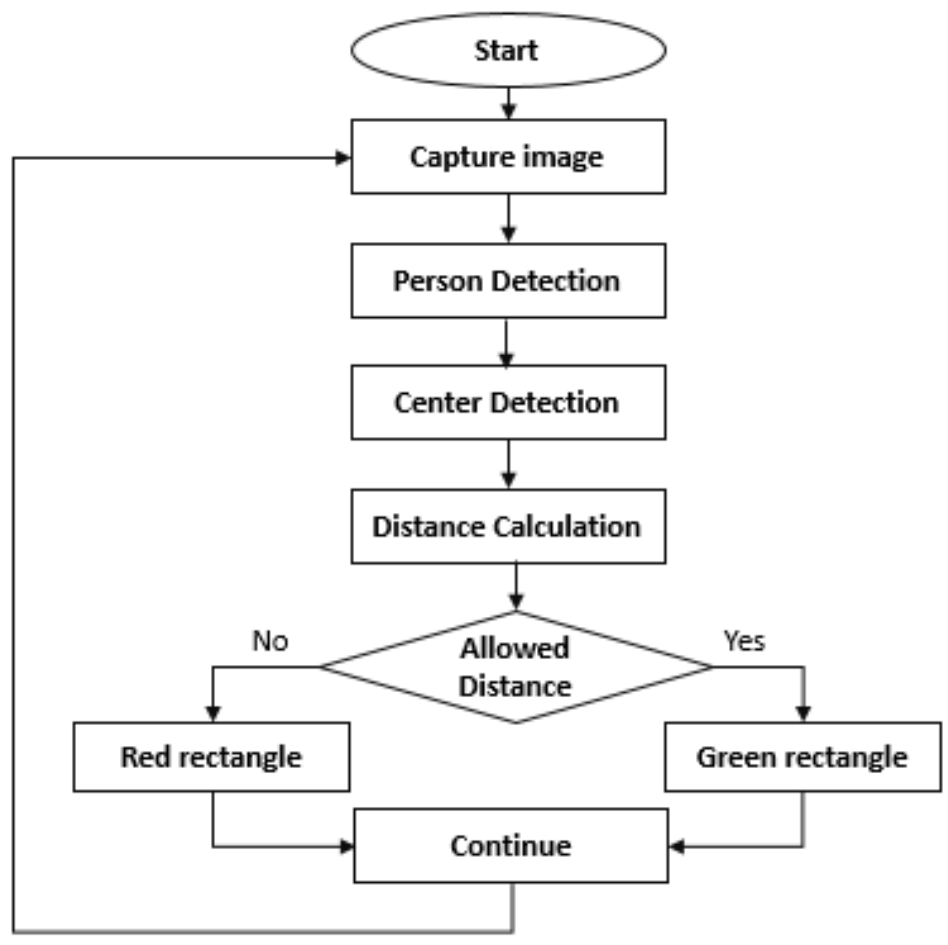

Figure 2. Block diagram for the proposed system

\section{Object Recognition}

Image classification concept refers to assign each image to its class label, whereas object localization refers to bound the detected object by rectangle, these two challenges lead to new concept called object recognition, in real, many complex tasks are cooperated for identifying object recognition.

If we used object recognition strategy in deep learning, so we should think of these algorithms:

- R-CNN and their variants, including the original R-CNN [9], Fast R- CNN [10], and Faster R-CNN .

- Single Shot Detector (SSDs).

- You Only Look Once (YOLO) [11].

However, R-CNNs head for high accuracy, but major drawback with the R-CNN is extremely slow, in general, single-stage detectors tend to give fewer accuracy than two-stage detectors but are meaningfully faster, YOLO is a good choice for a single stage detector, which is able to achieve a large number of object detections by execution combined training for both object detection and classification.

\section{YOLO}

YOLO (You Only Look Once) is a state of art Object Detector which can achieve object detection in real time with a very good accuracy. The first versions of YOLO have released in 2016, 2017 and 2018 (YOLO1 [11], YOLO2 [12] and YOLO3 [13]), later, within few days, another three versions have released (YOLO4 [14], YOLO5 [15] and PP-YOLO [16]). YOLO can make accurate detection for different objects like (persons, cars, animals, teddies, trees and others), in this research, YOLO4 is used to detect persons. 


\section{Dataset}

The COCO Object Detection Task is considered to rise the state of the art in object detection forward, dataset holds pictures of 91 objects, with a total of 2.5 million labeled instances in $328 \mathrm{k}$ images. COCO structures two object detection ways first by using bounding box output and the other object segmentation output [17], in this research, bounding box technique is used.

\section{Detects Full-Fledged Social Distancing}

In the previous step, $\mathrm{COCO}$ data set is used to make training with object recognition using YOLO4, while the video datasets that used to experiment the system are Virat dataset [18] and so, the people are detected in each frame video and bounded with boxes, by using OPENCV, Compute the Euclidean distance among centers of people and compare the distance with minimum allowed distance, then color the box of accepted distance with green, and the forbidden with red.

\section{Experimental Results}

The system is modeled using python, the code is taken from [19] and modified by using YOLO4 instead of YOLO3, the code is tested using Virat video dataset [20] and EPFL video dataset [21], to ensure if it can work well using online camera with different distances position of camera, the proposed system has worked well, and shows very satisfy results in online persons detection and social distance detection as shown in figures 3 and 4 below:

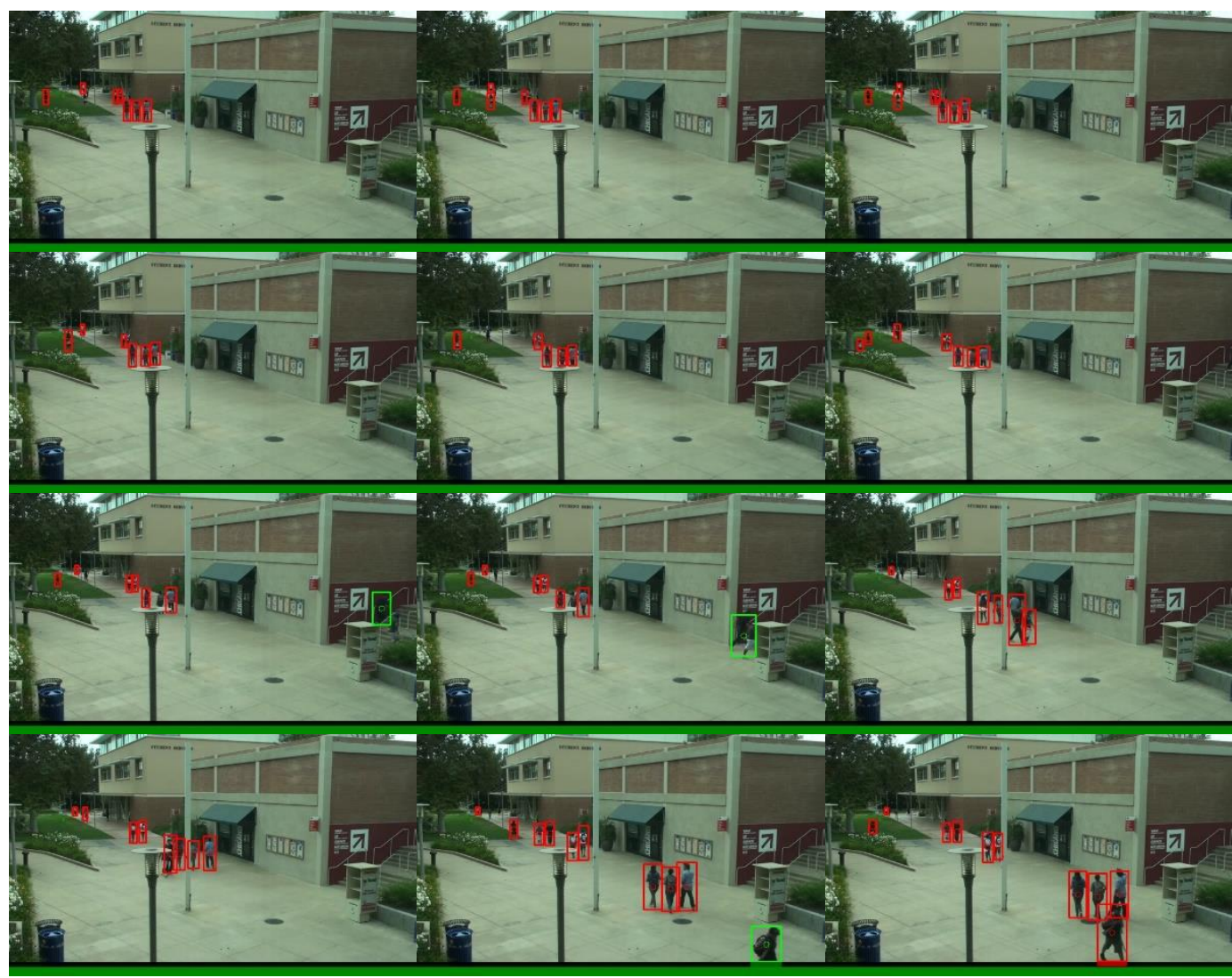

Figure 3. Frames of Virat video 


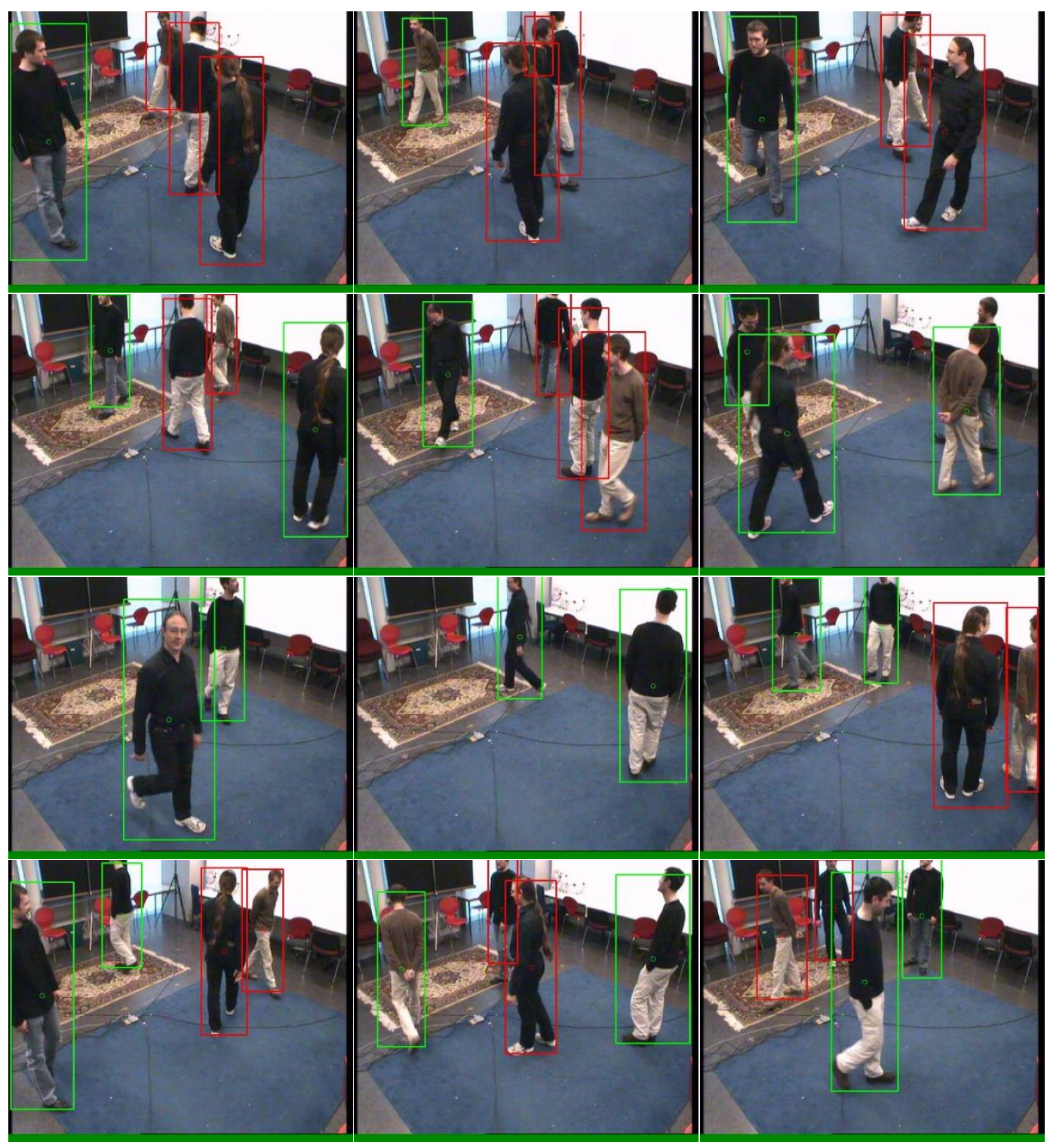

Figure 4. Frames of EPFL video

The position of camera and the distance between persons and camera are different, and make different results in detection, so two videos are used with different positions, as shown in table1:

Table1. details of tested system

\begin{tabular}{|l|l|l|}
\hline \multicolumn{1}{|c|}{ Database } & \multicolumn{1}{c|}{ Camera distance } & \multicolumn{1}{c|}{ On System } \\
\hline Virat & $10 \mathrm{~m}-20 \mathrm{~m}$ & 140 Pixels \\
\hline EPFL & $5 \mathrm{~m}-3 \mathrm{~m}$ & 50 Pixels \\
\hline
\end{tabular}

\section{Conclusion}

The system is implemented and tested using two different videos with different distances, and shows very satisfied results, by tuning the distance in system. The system can be used in schools, malls, streets and any other place may be crowdy with people. The system gives real time detection for video stream. 
The limits of this work, still, direction of face doesn't involve in the acceptance distance, where if the two adjacent persons are in forbidden distance but their faces are opposite, so the distance should be accepted however each one in the opposite direction.

\section{References}

[1] "World Health Organization." https://www.who.int/.

[2] K. Prem et al., "The effect of control strategies to reduce social mixing on outcomes of the COVID-19 epidemic in Wuhan, China: a modelling study," Lancet Public Heal., vol. 5, no. 5, pp. e261-e270, 2020, doi: 10.1016/S2468-2667(20)30073-6.

[3] M. J. Siedner et al., "Social distancing to slow the US COVID-19 epidemic: Longitudinal pretest-posttest comparison group study," PLoS Med., vol. 17, no. 8 August, pp. 1-12, 2020, doi: 10.1371/JOURNAL.PMED.1003244.

[4] S. Yadav, "Deep Learning based Safe Social Distancing and Face Mask Detection in Public Areas for COVID-19 Safety Guidelines Adherence," Int. J. Res. Appl. Sci. Eng. Technol., vol. 8, no. 7, pp. 1368-1375, 2020, doi: 10.22214/ijraset.2020.30560.

[5] N. S. Punn, S. K. Sonbhadra, and S. Agarwal, "Monitoring COVID-19 social distancing with person detection and tracking via fine-tuned YOLO v3 and Deepsort techniques," pp. 1-10, 2020, [Online]. Available: http://arxiv.org/abs/2005.01385.

[6] S. Ramadass, L., Arunachalam, S., Z., "Applying deep learning algorithm to maintain social distance in public place through drone technology," Int. J. Pervasive Comput. Commun., vol. 16, no. 3, pp. 223-234, 2020, [Online]. Available: https://doi.org/10.1108/IJPCC-05-2020-0046.

[7] A. M. M, F. F. Alkhalid, and B. K. Oleiwi, "Online Blind Assistive System using Object Recognition," vol. 3 , no. 12 , pp. $47-51,2019$.

[8] R. Keniya and N. Mehendale, "Real-time social distancing detector using SocialdistancingNet- 19 deep learning network."

[9] R. Girshick, J. Donahue, T. Darrell, and J. Malik, "Rich feature hierarchies for accurate object detection and semantic segmentation," Proc. IEEE Comput. Soc. Conf. Comput. Vis. Pattern Recognit., pp. 580-587, 2014, doi: 10.1109/CVPR.2014.81.

[10] R. Girshick, "Fast R-CNN," Proc. IEEE Int. Conf. Comput. Vis., vol. 2015 Inter, pp. 1440-1448, 2015, doi: 10.1109/ICCV.2015.169.

[11] J. Redmon, S. Divvala, R. Girshick, and A. Farhadi, "You only look once: Unified, real-time object detection," Proc. IEEE Comput. Soc. Conf. Comput. Vis. Pattern Recognit., vol. 2016-Decem, pp. 779-788, 2016, doi: 10.1109/CVPR.2016.91.

[12] J. Redmon and A. Farhadi, "YOLO9000: Better, faster, stronger," Proc. - 30th IEEE Conf. Comput. Vis. Pattern Recognition, CVPR 2017, vol. 2017-Janua, pp. 6517-6525, 2017, doi: 10.1109/CVPR.2017.690.

[13] J. Redmon and A. Farhadi, "YOLOv3: An Incremental Improvement," 2018, [Online]. Available: http://arxiv.org/abs/1804.02767.

[14] A. Bochkovskiy, C.-Y. Wang, and H.-Y. M. Liao, "YOLOv4: Optimal Speed and Accuracy of Object Detection,” 2020, [Online]. Available: http://arxiv.org/abs/2004.10934.

[15] "YOLOv5." https://models.roboflow.com/object-detection/yolov5.

[16] X. Long et al., "PP-YOLO: An Effective and Efficient Implementation of Object Detector," 2020, [Online]. Available: http://arxiv.org/abs/2007.12099.

[17] T. Y. Lin et al., "Microsoft COCO: Common objects in context," Lect. Notes Comput. Sci. (including Subser. Lect. Notes Artif. Intell. Lect. Notes Bioinformatics), vol. 8693 LNCS, no. PART 5, pp. 740-755, 2014, doi: 10.1007/978-3-319-10602-1_48.

[18] S. Oh et al., "AVSS 2011 demo session: A large-scale benchmark dataset for event recognition in surveillance video," 2011 8th IEEE Int. Conf. Adv. Video Signal Based Surveillance, AVSS 2011, pp. 527528, 2011, doi: 10.1109/AVSS.2011.6027400.

[19] A. Rosebrock, "OpenCV Social Distancing Detector." https://www.pyimagesearch.com/2020/06/01/opencvsocial-distancing-detector/.

[20] "Virat Video Dataset." viratdata.org.

[21] “EPFL Video Dataset.” https://www.epfl.ch/labs/cvlab/data/data-pom-index-php/. 